\title{
Engineering Management Framework to Achieve Safety of a Service of an Organization Based on Social Acceptance
}

\author{
Yuichi Otsuka $^{1^{*}}$, Hiroshi Noguchi ${ }^{2}$ \\ ${ }^{1}$ Top Runner Incubation Center for Academia-Industry Fusion, Nagaoka University of Technology, \\ Niigata, Japan \\ ${ }^{2}$ Faculty of Mechanical Engineering, Kyushu University, Fukuoka, Japan \\ Email: ${ }^{*}$ otsuka@vos.nagaokaut.ac.jp
}

Received November 13, 2011; revised December 19, 2011; accepted December 28, 2012

\begin{abstract}
We aim at a management framework of an organization in order to achieve its safety in a limited case for a system involving the organization for the service, its users and the surrounding society. The proposed framework is possible to allow safety managers to specify tasks involving safety measures based on the concept of "a study of safety". In the beginning, we discuss the definitions of safety and acceptance, which will be the target of safety management. Next, problems in which the hierarchy of components in the safety systems involves are noted. We then propose the following details of the management framework to achieve the safety of the service of the organization based on acceptance by the surrounding society. Applications of the components in the proposed framework are effectively demonstrated. The shown application can aid in visualizing a way of specification of the proposed concept in a target system.
\end{abstract}

Keywords: Safety Theory; System Management; Organizational Safety; Incident Report; Safety Rule; Hierarchy

\section{Introduction}

The extents of accidents within systems have become more serious according to the increases in complexities of the systems [1]. In a hierarchical structure composed of humans, the structure amplifies the effect of human errors by its complexity and finally results in an organizational accident. As a precaution to prevent a serious accident, the safety management involving a reduction in the occurrence of errors and the detection of errors before resulting in damage to the victims is indispensable. For the former factor, the concepts of human factors and standard safety measures for them have been studied $[2,3]$. Regarding the concept of the human factors, human errors emerge due to various causes in their workplace. A safety management system should then be constructed on the basis of the considerations for cognitive and physiological characteristics of humans. Considering the latter factor, the concept of a man-machine system [4] to optimize the interfaces between the both has recently been investigated. Furthermore, the concept of complexity adaptive system [6] based on the theory of complexity [5] has been proposed. This concept is applied to some cases in designing safety systems for patient safety management [7]. In other words, the activity for establishing the constructing policy of

"Corresponding author. safety systems is in progress.

Murakami has proposed a "safety theory" [8] as the universal concept of safety. He argued that safety itself is a value and that the "safety theory" should become metascience integrating normal safety engineering and social science. Axelrod [6] also showed the management framework of a complex organization whose purpose is not limited in achieving safety. On the other hand, Tominaga [10] discussed his theory of structural variation in which an observer within the system can organize the force of structural variation. This point indicates the necessity of including the interactions in workplaces (among workers practicing safety rules) in the process of achieving safety of the system. However, the framework in which the view of utilizing this bottom-up interaction is involved has not been established.

The concept of the "safety theory" normally includes the following elements.

1) The design concept of reliability and safety engineering including fail-safe and foolproof characteristics $[8,9,11]$.

2) Incident reporting $[2,12]$.

3) The concept of safety climate $[13,14]$.

4) Risk communication $[8,15]$.

There are many examples of the elements such as safety audits in nuclear plants [16] or marine plants [17], an incident reporting system in aviation safety [18] and the 
investigation for cognitions of risks in nuclear plants [15]. An integrating framework of these elements to establish a safety management system is necessary in the case where someone applies the above concept to a practical organization.

Horii et al. [19] have reported a concept of social techniques for safety involving visualization technique to the structure of the problems and handling the situations of society in order to take countermeasures for the problems and evaluate those effects. Their study targets involved various fields, and they reported remarkable results in handling the complex structure, such as the structure of emerging anxiety in humans due to the risks of nuclear plants [19]. Their major target was to enclose the problem and unfortunately the management framework for the systems as risk sources was little considered. If the integrating concept based on the above elements to specify the framework of the "safety theory" under limited conditions is established, it is possible to construct an effective and applicable framework for safety management systems in practical fields. However, to the author's knowledge, no investigation has been reported for an integrating concept.

This paper describes an engineering management framework for safety systems based on social acceptance for the limited target including an organization providing service, service users and the surrounding society. The proposed framework allows the specification of the safety management system in one application to become easier. All of the elements in the proposed framework are provided as examples, in order to refer to the examples in applications for other systems. Finally, we discuss the consequence of our proposal for safety system management.

\section{Definitions of Safety and Acceptance}

At first, definitions for conditions of safety and acceptance are discussed using the simplest case shown in Figure 1.

These conditions emerge from the relationship between two subjects in the figure.

Service subject: A subject who creates a service and serves it for users.

User object: A subject who purchases the service or considers utilization of it.

In the case where the service is one product, the service subject is the designer of service and the user object is regarded as a customer.

The service includes both its quality [20] and risk. The involved risk may determine the expected damage to the users. This relation can determine the condition for safety $[9,11]$.

Safety: The extent of the risks involved in the service remains lower than the target level ( $D_{\text {target }}$ in Figure 1).

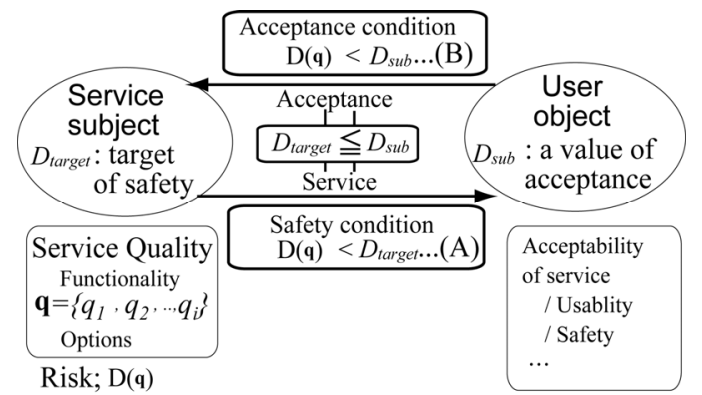

Figure 1. Definition of safety of service and acceptance.

The definition is based on the assumption that risk can be calculated by the function of probability of an occurrence and the extent of damage [9].

On the other hand, Slovic revealed that the subjective risk that people perceive is different from the value of objective risk calculated by statistical data [21]. This fact requires that the target of safety is also lower than the value people accept. Consequently. the definition of acceptance is shown as follows.

Acceptance: The extent of the risks involved in the service remains lower than the level acceptable to the user objects ( $D_{\text {sub }}$ in Figure 1).

"Sense of security" has meaning similar to that of "acceptance". However, this term may lead people to misunderstand that no risk is involved, which cannot be achieved by any activities. The term "acceptance" is preferably used because it means that the user objects autonomously judge the acceptability of a service by considering both its quality and its risk. The term "active security" is also proposed [19]; however, it may have the same meaning as "acceptance".

The service subject provides a service to the user objects under the constraint condition $D_{\text {target }} \leq D_{\text {sub }}$. To specify the values in the equation, a proactive risk communication must be practiced.

\section{Considerable Aspects Emerge from the Hierarchies of Subjects to Achieve Safety in Social Acceptance}

The service subject becomes a hierarchical organization when he produces and provides a complex service [5]. This hierarchy may produce considerable new aspects as shown in Figure 2 on the process to achieving safety.

\subsection{Aspects Produced by the Hierarchy of an Organization Creating a Service}

We discuss the problems in a safety management process using a simple hierarchical structure of an organization, such as Safety manager-section manager-worker, shown in Figure 2. The organization involves the following problems in safety management activities. 


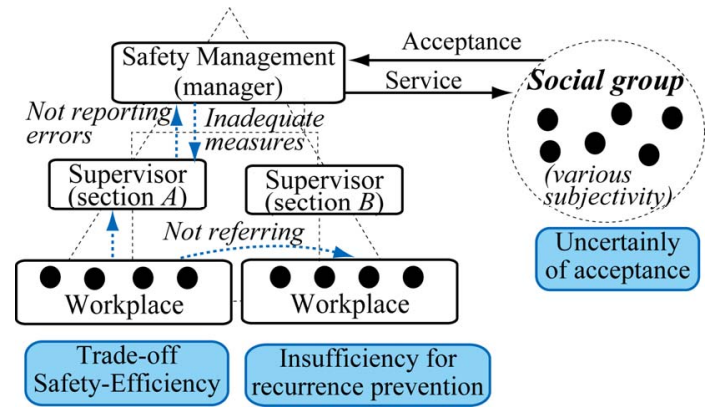

Figure 2. Considerable problems produced by the hierarchy of the service subject and the user object.

\subsubsection{Problems Involved in the Relationship between a Section Manager and Workers}

1) A section manager has difficulty in observing a detailed (complex) condition in his workplace. He/she often misunderstands that problems in his workplace are simply caused by errors of the workers. Furthermore, the section manager also orders the workers to obey current safety rules which have not properly adapted to the working situations. These safety rules may be a significant workload for the workers, and the ambivalent relation between safety and efficiency then becomes a considerable problem.

2) Workers often hesitate to report their errors to a safety management section. This problem forces the safety manager to handle the real tendency of errors occurring in the workplaces and to analyze the causes of the errors. This also damages the effectiveness or the acceptability of safety measures proposed by the safety manager. Namely, safety measures determined based on insufficient information may involve difficulty in their practical efficiency in real workplace. The safety rules may increase their load in practice and produce many more errors or violations.

\subsubsection{Problems Involved in the Relationship among Sections}

Workers are unable to judge whether their workplaces are objectively safe, because they have no target for comparison. This problem misleads workers into confusing which practices in the workplace are really effective for process safety. Furthermore, even if they collect numerous error reports, they cannot perceive them as an alarm that tell them the necessity of taking safety measures. Because the errors are a usual event for them without objective data. To make matters worse, these reports including real errors by workers are often hidden by a section manager, because he she may consider that these reports are not very useful for neighboring sections.

\subsection{Aspects Produced by the Hierarchy of User Objects: As a Social Group}

A user object in Figure 1 has a single personal view of an evaluation. The constraint condition for safety-acceptance is then determined by his individual evaluation. However, a social group, composed of various persons, is a target as customers of the organization. The social group has no autonomous subjectivity other than a set of individual ones among its members. The organization then has the problem.

\subsection{Policies of Treatments for the Considerable Aspects}

In summary, the following treatment should be necessarily investigated in order to achieve safety of a service based on social acceptance, as illustrated in Figure 2.

1) Establishment of a predicting method for a target of social acceptance (if possible, a quantitative prediction is preferable).

2) Constructing an improvement process for safety rules using incidents or occurrence reports.

3) Constructing an improvement method for acceptability of safety rules by treatment of worker's perception to lessen the ambivalent relation between the safety of service and the efficiency of the service.

\section{Management Framework to Achieve Safety of a Service on the Basis of Social Acceptance}

Figure 3 shows the proposed management framework to achieve the safety of a service of an organization based on social acceptance. The proposed framework includes the following participants.

Organization: Hierarchical structure by Safety Manager-Supervisor-Worker to provide a service for users.

Users group: A set of persons who have accepted the quality of the service and use it.

Surrounding society: A society of persons who including both the organization and the users group. A competitive organization providing the same services can also be existed.

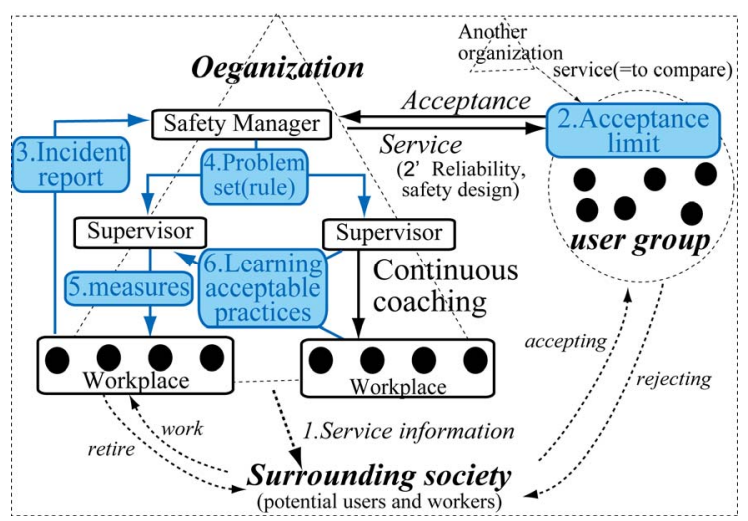

Figure 3. Management Framework to achieve the safety of a service by an organization based on acceptance by a society. 
We now discuss the procedure of operating the proposed framework as the follows.

1) Communicating the quality of a service to the surrounding society.

2) Determining the acceptance limit for the quality of service by a society and transforming it into an organizational safety target. (Reliability and safety design, manufacturing and provision of a service under the safety target)

3) Establishing a system for collecting failure information (incidents or adverse events) inside the organization.

4) Visualizing the problems in the safety systems based on the collected incidents.

5) Introducing the safety measures for the problem by a safety manager.

6) Improving the contents of the safety rules to optimize the complex situations in the workplace by comparing the execution method among sections

\subsection{Determining the Acceptance Limit for the Quality of Service by a Society (Processes 1-2 in Figure 3)}

The attitude of a person for a service of an organization is determined by a judgment based on service information, as shown in Figure 4. We simply assume that the status of persons involves only two conditions, accepting or rejecting. The status of persons can be changed by the interactions (discussions) among persons [22]. However, if a target set for the quality of service is lower than one characteristic value in the organization, the ratio of accepting person among total persons can be stable after a sufficient number of interactions [23,24]. This value is the acceptance limit of the society for the quality of the service. The organization should determine its safety target in order to satisfy the acceptance limit. This service should be continuously accepted by the society to earn a sufficient profit; otherwise the organization will be bankrupt.

\subsection{Organizational Learning from the Collected Incidents (Processes 3-6 in Figure 3)}

The process of achieving the safety target determined by the society is shown in Figure 5. Firstly, a collecting system for failures in the workplaces must be established. Incident reports $[2,18]$ are normally collected. In the introduction stage of the incident report system, it should be necessary to evaluate the usability of the system in order for users to accept comfortably [25]. Next, the collected data must be analyzed to investigate the cause and determine the problem in the organization. The kinds of problems are considered such as human factors as well as technical and management problems [27]. Among these three types of problems, the organization should take measures for the management problem (especially relating the safety rule and the management systems) because this

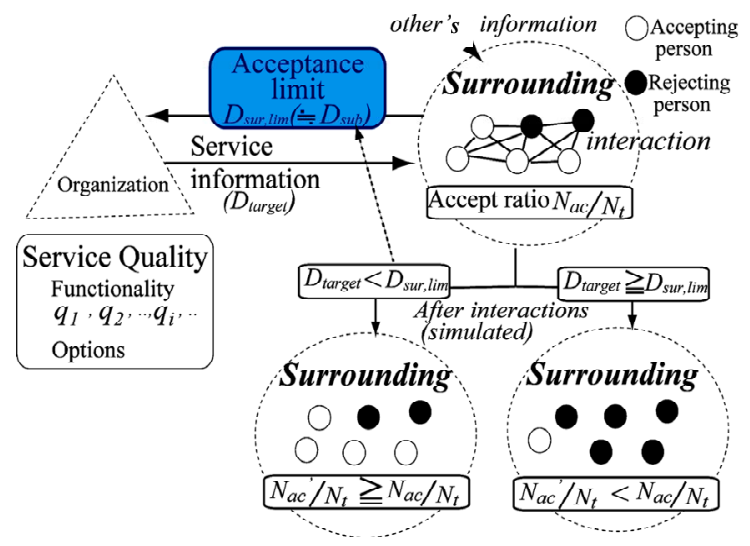

Figure 4. Determination of safety target of service by the predicted acceptance limit of the surrounding group.

causes technical and human errors to occur. The reported tendencies involved in the determined problems can be used to visualize the potential problems in the management systems. If no measures have been introduced, the safety manager should call the experts to analyze the cause and take safety measures in the workplaces.

In introducing the safety measures, there are two cases to be considered, such as the case of known measures or new measures. The new measure should have no difficulty in execution. In the latter case, the already practiced measures were probably insufficient. To improve the measure's effectiveness, the details in the measure should include consideration of them to the complexity in the workplace. It is possible to improve the contents of the safety measures in order to adapt for the complexity by collecting practices in the workplaces and extracting its "good" heuristics. In this process, by introducing the practices of the safety rules, the details of the safety rules can be improved sufficiently to attain both work efficiency and the worker's acceptance. These continuous treatments enable a safety manager to establish the effective management framework in which occurrences of human errors can be reduced and damage to victims may also be prevented by the certain detecting the errors.

\subsection{On Evaluating the Framework of Learning from Failures}

The evaluation of safety measures can be quantified by the extent to which they decrease the occurrences of errors. However, the evaluation cannot be avoided based on the failure information that workers voluntarily report. The case in which the change in the number of reports does not correspond to the change in the practical number of error occurrences is naturally predicted. If the safety manager decides that a lower number of reports is good for organizational safety, the workers will more hesitate to report, because the activity of not reporting will obtain "good" evaluation by the manager. In this case, the differ- 
ence between the number of reports and that of the practical occurrences should be extended, and effectiveness of the evaluation based on the number of reports will be lost. Therefore, an indicator which can encourage reporting by workers and express the decrease in damage due to errors is necessary.

Continuously introducing safety measures in the workplace may yield difficulty in determining the effect of individual measures. However, it is not really necessary to identify the effect of one safety rule or measure. Entire evaluation of the whole framework shown in Figure 5 can be performed using the indicator mentioned above. Furthermore, in the process of introducing safety measures, the supervisors in one workplace probably participate. Their judgment based on experiences in a complex situation in the workplace can prevent bad measures from being introduced.

\subsection{Introducing the Concept of Acceptable Safety Rule}

The contents of safety rules must be kept by workers. In order to obtain the worker's acceptance, consideration for increasing the conscious effort to keep the rule is necessary. Rule violating action is affected by both individual factors and organizational factors. In Figure 5, the objective analysis by experts and practical heuristics of workers can improve the contents of safety rules to become acceptable and safe. By participating workers themselves into the improvement process, the improved rule can be better acceptable by the workers because of explicitly considering both the factors for rule violating actions [29].

The flow of the work above mentioned is continuously practiced up to achieving the target of safety. It should be noted that the target itself may be changed according to a change in the situation of a society. This point will require a safety manager to update the safety target in a timely manner.

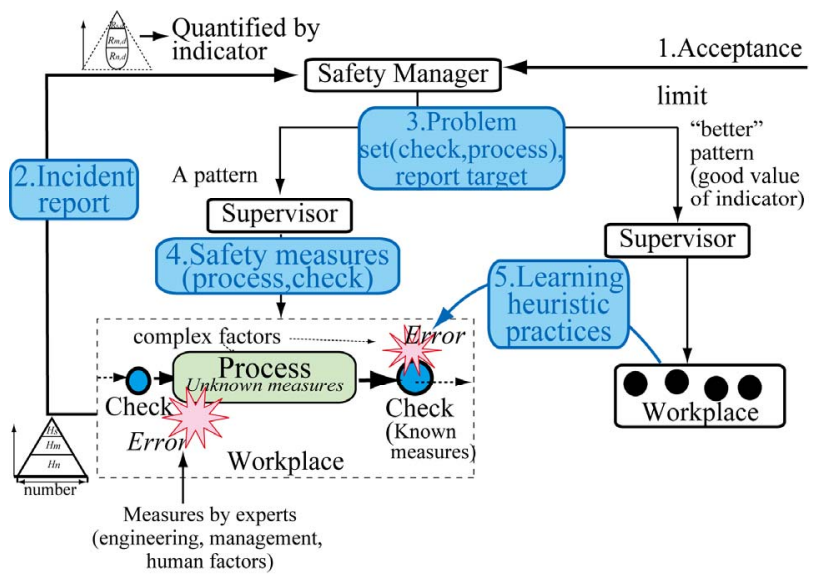

Figure 5. Safety management system to achieve safety and accepted rules.

\section{Case Studies for Elements in the Proposed Framework}

We provide example cases in engineering and patient safety fields for each element in the proposed framework to support specification of the proposed framework.

\subsection{Quantification of the Target Quality of Service: Prediction of the Acceptance Limit Cost}

Otsuka et al. [24] reported that the state of acceptance for a social group is that the ratio of accepting person for one service can be maintained after sufficient interaction (discussion) among persons. An acceptance limit cost is also defined as the maximum cost of the acceptance. Furthermore, the prediction method for the value of the acceptance limit by combining simulations using cellular automata and experiment with real persons to determine the value of control parameters in the simulation. For example, the prediction of airplane fares shown in Figure 6 is conducted. The predicted value is accepted for almost all examinees at that time. Using the prediction method and modeling the interaction process among humans for one group can quantify the acceptance limit of a service. The proposed method is also applicable to predict the extent of acceptable safety by one society.

\subsection{Development of Incident Reporting System for Enthusiastic Reporting}

A web-based incident reporting system has been developed in the patient safety field [25]. In the development stage of the system, the results of usability tests by real workers which indicate a better functionality of the system than that of a previous one may be effective to allow workers to use new system. After introducing the developed system, a number of reports increased more than $50 \%$ after two years, which indicates efficiency improvement in collecting failure information. This research can

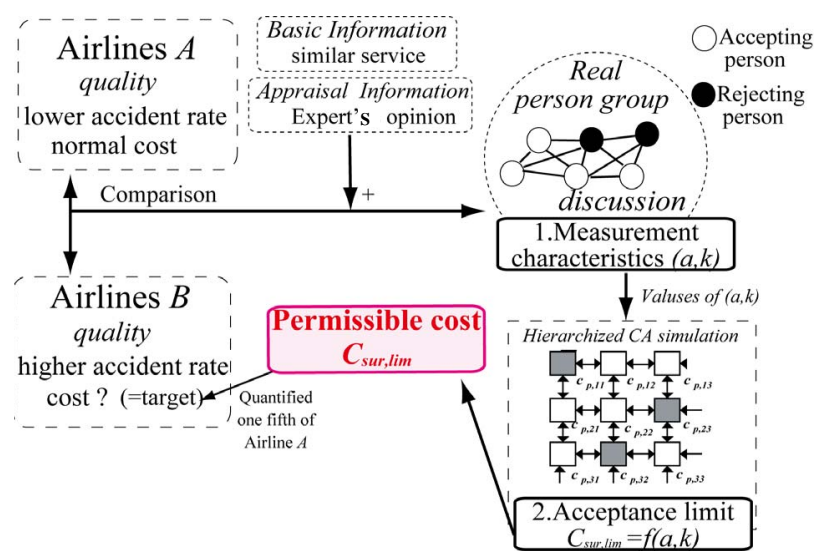

Figure 6. Case study of predicting acceptance limit; permissible cost of airlines fares [24]. 
be a reference in establishing an acceptable reporting system for practical workers.

\subsection{Pattern Indicator for the Damage Distribution of Incidents for Evaluating the Entire Framework}

The pattern indicator for the damage distribution of incidents shown in Figure 7 was proposed in order to express both the increase in reports and the decrease in the extent of damages [26]. The proposed indicator is defined by the ratio of the number of reports for unacceptable damage among the total reports. Figure 7 illustrates a predicted correlation relationship between the proposed indicator and the average numbers of reports for unacceptable damage in the workplaces. The predicted relationship can be observed in the incident data from one university hospital [26]. Using Figure 7 allows the safety manager to quantitatively evaluate the effectiveness of the entire safety management framework by the changes in the value of the pattern indicator.

The vertical axis in Figure 8 can be regarded as the indicator of safety. If the safety target in the vertical axis is determined, the target value of the horizontal axis is also determined using the regression line. Therefore, Figure 7 can transform the safety target externally determined into an internal reporting target for the management sections.

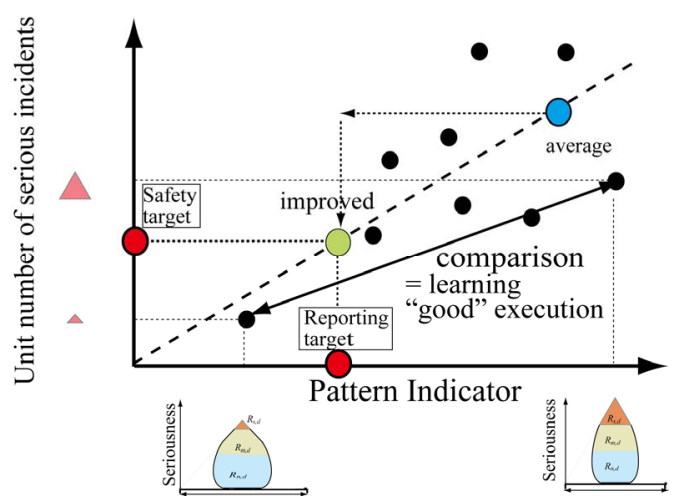

Figure 7. Determination for comparing sections by pattern indicator [26].
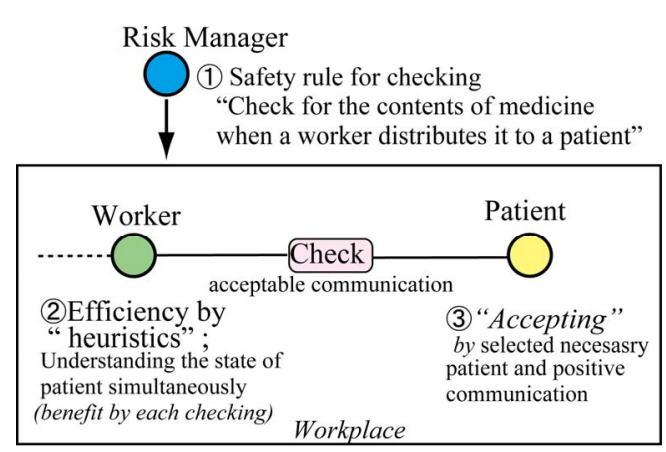

Figure 8. Example of acceptable safety rule; checking with patient by communication [27].

\subsection{Improving Procedure for the Contents of Safety Rules Using Incident Reports}

By the analysis of collected incidents, causes of the incidents relating to fails in checking, work process and others were determined [27]. The safety manager decided to select one of the causes, "the failure in checking the contents of drugs in distributing to patients" as one target of improving. Comparisons between sections using the pattern indicator and interviewing supervisors in the sections could lead to an acceptable safety rule based on practical heuristics, as shown in Figure 8. The resulting rule involves the following considerations in order to satisfy all participants.

Supervisor: The contents of the check safety.

Worker: Communicating to patients to handle their conditions at the same timeof checking for efficiency improvement.

Patient: Accepting for participation in the checking process by active communications from workers.

The framework in Figure 8 can be regarded as one target of safety rules to be achieved. Using the pattern indicator and comparing an empirical method for practicing safety rules in sections can enable visualization of those acceptable safety rules.

\subsection{Job Training Concept in Design Review Process; Example of Acceptable Safety Rule in Engineering Management}

Figure 9 shows the job training concept in a design review process, which is named Design Review Based on Failure Mode (DRBFM), proposed by Shimizu et al. [28]. Safety and acceptance by all participants can be achieved by the following.

Manager: Establishing the process of DRBFM for precautionary prevention (Safety).

Professional adviser: Introducing his knowledge and experience to designers in the design review process (Efficiency).

Designer: Determining his lack of knowledge through by discussion with the adviser (Acceptance).

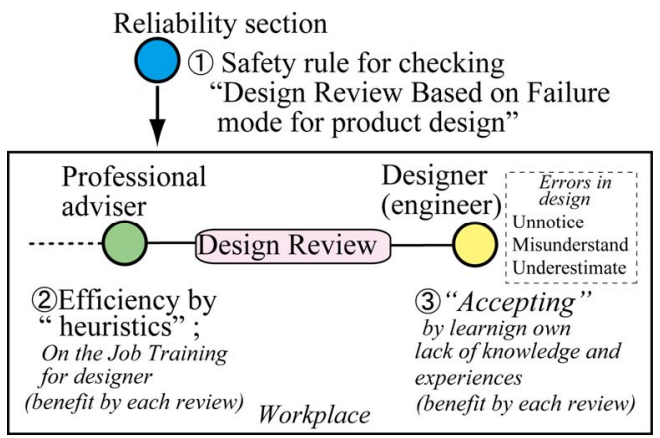

Figure 9. On-the-job training in design review based on failure mode [28]. 
The type of the framework shown in Figure 9 is obviously the same as that in Figure 8. This similarity can support the applicability of the concept of an "acceptable safety rule" for workers not only for patient safety fields but also for other fields such as engineering management. Consequently, the target of the safety management framework is to improve the contents of the safety rules to become an "acceptable safety rule" for workers in order to achieve safety target.

\subsection{Applicability of the Entire Framework}

Table 1 shows the correspondence between the elements of the proposed framework and the explanatory examples. All elements in Figure 3 can be specified in a practical management system because all of the elements possess the correspondent examples. The functionality of the entire framework should be discussed. The difficulty in the connection between the second element and the third element in Figure $\mathbf{3}$ is concerned, because these elements were applied to different fields. However, both processes are controlled using the values in the axes in Figure 8.

We can then integrate the considered connection successfully using Figure 8. This is because Figure 8 can be observed in a practical subject and can be utilized effectively for safety management. Therefore, the proposed framework is totally effective for achieving one safety target and possessing a sufficient applicability to practical subjects.

\section{Discussion}

Workers should always obey one safety rule, because they cannot predict when they take an error [30]. In some situations, an ambivalent relation between safety and efficiency of the work probably becomes a considerable problem, as illustrated in Figure 10 [30]. Namely, a worker may judge whether he keeps one safety rule by comparing the loss due to the work load with the benefit of preventing future accident using the rule. It is very easy for all workers to perceive the work load. Unfortunately, cognition of the benefit due to the rule greatly varies according to the subjectivity of the workers [8]. The less cognizant the person is of the benefit of a safety rule, the more likely the person will not keep the rule. The ordinary treatment for this assignment achieves only a not-specific result, "recommendation for obtaining the consciousness of prior thinking for safety".

The proposed management system model is a kind of specified adaptive complex system model $[6,7,31]$ to achieve safety of a service produced by the system. The roles of agents (workers, a supervisor and a manager) are determined, subject to the respective hierarchy of the system structure. Heuristic practices will be examined by the safety management activity for the purpose of improving safety, efficiency and the acceptability of the safety rules. These bottom-up activities will change the internal (safety) rules in the organization, which means that the structure of the system will have been slightly changed [10]. The described model in Figure 10 integrates internal (heuristic) experiences and external objective knowledge to achieve the condition of acceptable safety. This concept also corresponds to the internal interaction model in normal system theory [10]. This coherence supports the applicability of the proposed framework to other practical fields.

Table 1. Relationships between elements of the proposed management framework and applications in engineering safety and patient safety.

Element of the proposed framework

1. Service information

2. Acceptance limit

(2’: Reliability and Safety design for the service, manufacturing and serving)
Application 1: Engineering Safety

Risk and time for an airplane [24]

Airplane fare [24]
Web-Based report system [25]

Factors in safety rules for checks or specific works [27]

Monthly report of the incidents to workers
5. Safety measures

6.Learning acceptable measures
On-the-job training in DRBFM processes [28]
Heuristic practices in workplaces [27] found by using the pattern indicator [26] 


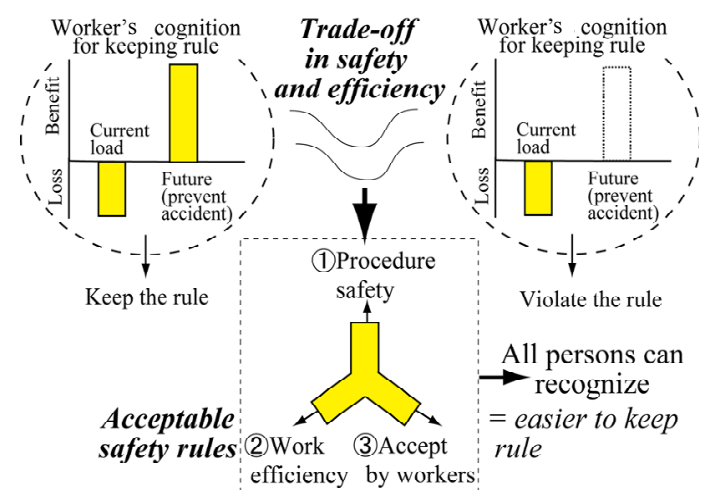

Figure 10. Concept of changing trade-off relationship for acceptable safety work.

The elements in the proposed framework have undergone empirical case study in the field of patient safety management or engineering management, which indicates sufficient applicability of the proposal to practical targets. Furthermore, the concept of acceptable safety rules shown in Figure 10, as one of the desirable targets of improving the quality of safety rules, can be specified in both fields. This strongly indicates the possibility of establishing an acceptable safety rule in other fields. In the process of application, the total guidance that means the normal structure of the management framework is given in Figure 3. In addition, when practitioners are confused about how to establish each element in the normal framework, the case studies described in section 5 can help them to be successful.

This framework is effective only after the problems to be solved are discovered. However, the complex structure of the problems may hide their causes from analysis by safety management activities. In this case, a soft system model [32], a self-organized map or direction graphs [19] will help the safety manager to visualize the complexity and also reveal its causes.

\section{Conclusion}

We have proposed the concept of a management framework to achieve the safety of a service of an organization based on acceptance by a society. The proposed concept includes collecting failure information and learning from failures in order to improve the contents of safety rules to be acceptable and safe for workers. We have provided examples for all elements in the proposed framework. These examples can be useful in the case of application to other practical subjects.

\section{Acknowledgements}

One of the authors was partially supported by the Top Runner Incubation System through the Academia-Industry Fusion Training in the Promotion of Independent Research Environment for Young Researchers, MEXT, Japan.

\section{REFERENCES}

[1] T. Kletz, "Learning from Accidents," 3rd Edition, Gulf Professional Publishing, London, 2001.

[2] J. Reason, "Managing the Risks of Organizational Accidents," Ashgate Publishing Limited, London, 1997.

[3] J. Reason and A. Hobbs, "Managing Maintenance Error a Practical Guide," Ashgate Publishing Limited, London, 2003.

[4] B. A. Turner, "Man-Made Disasters," Wykeham Publications, London, 1978.

[5] L. Von Bertalanffy, "General Syetem Theory: Foundations, Development, Applications," George Braziller, New York, 1968.

[6] R. Axelrod and M. D. Cohen, "Harnessing Complexity: Organizational Implications of a Scientific Frontier," Simon \& Schuster Incorporated, New York, 1999.

[7] Institute of Medicine, "Crossing the Quality of Chasm," National Academy of Science, Washington DC, 2001.

[8] Y. Murakami, "Safety Theory," Seidosha, Tokyo, 1998.

[9] M. G. Stewart and R .E. Melchers, "Probabilistic Risk Assessment of Engineering Systems," Chapman \& Hall, London, 1996.

[10] K. Tominaga, "A theory of Action and Social System: Toward a Theory of Structure Function-Change," University of Tokyo Press, Tokyo, 1995.

[11] M. Mukaidono, "Mechanical System Safety Techniques in Globalization Period," The Nikkan Kogyo Shimbun Limited, Tokyo, 2000.

[12] C. D. Reese, "Accident/Incident Prevention Techniques," 2nd Edition, Taylor \& Francis, New York, 2001. doi:10.1201/9780203472101

[13] F. W. Guldenmund, "The Nature of Safety Culture: A Review of Theory and Research," Safety Science, Vol. 34, No. 1-3, 2000, pp. 215-257. doi:10.1016/S0925-7535(00)00014-X

[14] J. N. Sorensen, "Safety Culture: A Survey of the State-ofthe-Art," Reliability Engineering \& System Safety, Vol. 76, No. 2, 2002, pp. 189-204. doi:10.1016/S0951-8320(02)00005-4

[15] M. G. Morgan, et al., "Risk Communication: A Mental Model Approach," Cambridge University Press, London, 2002.

[16] S. Gunderson, "A Review of Organizational Factors and Maturity Measures for System Safety Analysis," System Engineering, Vol. 83, No. 3, 2005, pp. 234-244. doi:10.1002/sys. 20033

[17] L. Beltracchi, "Plant and Safety System Model," Reliability Engineering \& System Safety, Vol. 64, No. 2, 1999, pp. 317-324. doi:10.1016/S0951-8320(98)00071-4

[18] G. D. Edkins, “The Indicate Safety Program: Evaluation of a Method to Proactively Improve Airline Safety Performance," Safety Science, Vol. 30, No. 3, 1998, pp. 275295. doi:10.1016/S0925-7535(98)00049-6

[19] H. Horii, "Social Techniques for Safety and Security," University of Tokyo Press, Tokyo, 2006.

[20] G. Taguchi, "Quality Engineering in Production Systems," 
McGraw-Hill, New York, 1988.

[21] P. Slovic, "The Perception of Risk," Earthscan Publication Limited, London, 2000.

[22] S. Wolfram, "A New Kind of Science," Wolfram Media Incorporated, Champaign, 2002.

[23] Y. Otsuka, T. Yoshimura and H. Noguchi, "Quantitative System Management with Hierarchized Cellular Automata," Transactions of the Japan Society of Mechanical Engineers Part C, Vol. 69, No. 688, 2003, pp. 3422-3430. doi:10.1299/kikaic.69.3422

[24] Y. Otsuka, J. Ayuzawa and H. Noguchi, "Quantitative Prediction for Acceptance Limit Value to Society for the Service Quality of an Independent System," Simulation Modelling Practice and Theory, Vol. 15, No. 9, 2007, pp. 1103-1119. doi:10.1016/j.simpat.2007.07.004

[25] Y. Otsuka, T. Abe, H. Noguchi and A. Makinouchi, "Development of Incident Report Database for Organizational Learning," Transactions of the Society of Instrument and Control Engineers, Vol. 45, No. 2, 2009, pp. 120-126.

[26] Y. Otsuka, J. Ayuzawa and H. Noguchi, "Proposal for a Quantitative Indicator for a Shape Pattern in a Damage Distribution of Incident Reporting and Its Utilization Concept," Transactions of the Japan Society of Mechanical Engineers Part C, Vol. 74, No. 740, 2008, pp. 251-259.
[27] Y. Otsuka, J. Ayuzawa, M. Akiyoshi and H. Noguchi, "Improvement Framework for Safety Rule Using Incident Reporting and Worker's Practical Heuristics (Empirical Consideration in Patient Safety Management)," Transactions of the Japan Society of Mechanical Engineers Part C, Vol. 74, No. 740, 2008 , pp. 260-267.

[28] Y. Otsuka, H. Shimzu and H. Noguchi, "Efficacious Design Review Process Including On-the-Job Training in Finding Misunderstand Errors," International Journal of Reliability, Quality and Safety Engineering, Vol. 16, No. 3, 2009, pp. 281-302. doi:10.1142/S021853930900340X

[29] Y. Otsuka, R. Misawa, H. Yamaguchi and H. Noguchi, "A Consideration for Using Workers' Heuristics to Improve Safety Rules Based on Relationships between Creative mental Sets and Rule-Violating Actions," Safety Science, Vol. 48, No. 7, 2010, pp. 878-884. doi:10.1016/j.ssci.2010.01.023

[30] A. R. Hale and P. Swuste, "Safety Rule: Procedural Freedom or Action Constraint?" Safety Science, Vol. 29, No. 3, 1998, pp. 163-177. doi:10.1016/S0925-7535(98)00020-4

[31] H. A. Simon, "Sciences of Artificial," 2nd Edition, Massachusetts Institute of Technology Press, Cambridge, 1981.

[32] P. Checkland and J. Scholes, "Soft Systems Methodology in Action,” John Wiley \& Sons Limited, Sussex, 1990. 